\title{
On the Statistical Evaluation of Dose-Response Functions *
}

\author{
A. M. Kellerer and J. Brenot** \\ Radiological Research Laboratories, Department of Radiology, \\ College of Physicians and Surgeons of Columbia University, New York
}

Received July 26, 1973

Summary. The linear-quadratic dependence of effect on the dose of ionizing radiation and its biophysical implications are considered. The estimation of the parameters of the response function and the derivation of the joint confidence region of the estimates are described. The method is applied to the induction of pink mutations in Tradescantia which follows the linearquadratic model. The statistical procedure is also suitable for other response functions.

\section{Introduction}

The study of dose-response relations has always been one of the main topics of radiation biology and radiation biophysics. Various theoretical models, many of them linked to hit- or target-theory, have been used to obtain analytical approximations for such relations. However, only few attempts have been made to test the fit of the experimental results to the analytical expressions. This lack of attention to the quantitative evaluation of dose-response curves may partly be due to the fact that the models usually employed have lost their original biophysical interpretation, since it has been shown that the processes of energy deposition by ionizing radiation are far more complex than postulated in hit- or target-theory $[11,12]$, and that the resulting analytical expressions are arbitrary special cases among a wide class of more general stochastic equations [6]. The lack of statistical treatment may also be due to the fact that equations such as the multi-target formula contain the unknown parameters in non-linear form and that therefore the established techniques of linear regression analysis cannot be readily employed.

Recently it has been pointed out that at least for certain cellular effects and in the range of small doses one deals with a simple linear-quadratic dose-response curve, and that the parameters in such response curves have well defined biophysical meaning [7]. Earlier the linear-quadratic relation had been invoked in the special case of the formation of dicentric chromosomes by ionizing radiations, and it has been extensively discussed in the literature (see e.g. $[8,16,1,10]$ ). In the following, statistical methods will be considered to obtain the least-squares fit and the confidence region for the parameters of a dose-effect relation. The linearquadratic model will be treated in detail as a particularly simple example and because of its special relevance to the important problem of the effects of ionizing

* Dedicated to Prof. Dr. O. Hug on the occasion of his 60th birthday.

** Present address: Commissariat à 1'Energie Atomique, DPr/Sps, Fontenay-aux-Roses, France. 
radiations at small doses. However, with minor modifications the considerations will be applicable also to other and more complicated cases.

\section{The Iinear-Quadratic Relation}

The remarks in this section do not concern details of the statistical procedure, they will, however, serve to put the statistical problem into perspective by outlining one area of particular applicability.

A systematic study of the relative biological effectiveness (RBE) of various radiation qualities as a function of absorbed dose has led to the conclusion that primary cellular damage produced by ionizing radiations in various eukaryotes is proportional to the square of the specific energy, $z$, in the nucleus or in sensitive sites of the nucleus of the order of one to several micrometer diameter [7]:

$$
\varepsilon(z)=k z^{2} \text {. }
$$

This relation indicates that the cellular damage is due to the interaction of pairs of radiation-induced sublesions or, more generally speaking, that the primary cellular damage results from a second-order reaction of radiation products. The quadratic dependence on specific energy leads to a linear-quadratic dependence on absorbed dose:

$$
\varepsilon(D)=k\left(\zeta D+D^{2}\right) .
$$

The linear term in this relation corresponds to that component of the damage which is due to lesions produced in one and the same particle track; $\zeta$ is the average increment of specific energy brought about by a single charged particle in the site. The quadratic term corresponds to the component of damage which is due to lesions produced by separate particles. Accordingly, one can state that the linear term represents the intra-track effect while the quadratic term represents the inter-track effect.

Before the microdosimetric concepts had been developed which lead to Eq. (2) the linear-quadratic dose dependence was deduced within the limits of the LET concept for the special case of the induction of dicentric chromosomes and centric rings. In this case $\varepsilon(D)$ stands for the mean number of these aberrations per cell. Lea [8] gives an excellent account of the reasoning which in essence corresponds to the more generalized microdosimetric arguments. Later there were numerous studies in which the ideas expressed by Lea have been applied and further developed (see e.g. [16, 1, 10]). It has been shown [7] that within the approximation of the LET concept Eq. (2) reduces to:

$$
\varepsilon(D)=k\left(22.9 \frac{\overline{L_{D}}}{d^{2}} D+D^{2}\right)
$$

where $\bar{L}_{D}$ is the dose average LET in $(\mathrm{keV} / \mu \mathrm{m}), d$ is the site diameter in $(\mu \mathrm{m})$, and $D$ is the absorbed dose in ( $\mathrm{rad}$ ). The relation expresses the fact that the chromosome aberrations are the result of two "single-breaks"; the linear term represents the yield of aberrations due to the interaction of two breaks produced by the same particle track, the quadratic term represents the yield due to the interaction of breaks which are produced by different charged particles.

It should be noted that these relations are only approximations even if one disregards saturation effects at higher doses. The quadratic dependence on energy 
concentration in the nucleus may not be strictly valid, and $\mathrm{Eq}$. (1) may contain an additional linear term in $z$. In the case of chromosome aberrations such a linear term could, for example, be due to the fact that some single-breaks are present even in unirradiated cells. But in this more general case one still obtains the linearquadratic dependence on absorbed dose; the only difference is that the ratio of the linear to the quadratic term is then larger than predicted on the basis of microdosimetric data or the LET value.

The linear-quadratic relation for the primary cellular damage has been deduced indirectly from studies of RBE. Because the observed experimental endpoint need not be linearly related to the primary cellular damage, one cannot always expect the simple linear-quadratic relation. Dose-effect curves for cytogenetic effects may therefore be particularly simple special cases. It has, however, been found that the growth reduction of Vicia jaba seedlings as a function of absorbed dose also agrees well with an expression which contains the linear and the quadratic term in dose $[4,5]$ :

$$
\ln G=\ln G_{0}-a_{1} D-a_{2} D^{2}
$$

where $G$ is the mean growth increment at dose $D$, and $G_{0}$ is the mean growth of the unirradiated plants. The same relation has been used to describe the survival of mammalian cells as a function of dose [14].

Fig. 1 gives results from a study in which the linear-quadratic dependence has been determined with high accuracy and down to very low doses for pink and colorless mutations in stamen hairs of Tradescantia ${ }^{1}$. The example in Fig. 1 is the yield of pink mutations obtained with $X$-rays in clone 02 [15]. For the purpose of the present discussion only the results at low doses will be considered; the plateau and the subsequent decline of the yield at high doses which may be due to cell killing will not be considered.

The full logarithmic plot has the advantage that one can judge the data accurately even in the range of low doses and low yields. In addition, this plot has the property that proportionality of the yield to a power, $D^{n}$, of the dose is indicated by a straight line of slope $n$. Thus, if one plots the yield minus the control incidence, the initial part of the curves with slope 1 corresponds to a linear dependence on dose while the part of the curves which approaches slope 2 at higher doses corresponds to a quadratic dependence on dose. In the following, these data will be analyzed on the basis of the linear-quadratic model expressed in Eqs. (2) and (3).

It is of interest to note that in cytogenetics the linear-quadratic model is sometimes substituted by the assumption of proportionality of the yield to a power of dose intermediate between 1 and 2. Depending on the observed value $n$ of the exponent the effect is then considered to be nearer to a linear or quadratic relation. It is obvious from Fig. 1 that such a statement has limited meaning; whether one finds a linear dependence, a quadratic dependence, or a dependence intermediate between these two cases, depends entirely on the dose range which is investigated. Frequently experimental studies cover only a narrow dose range, and it is then quite possible to approximate the results by a line segment with

1 Annual Report on Research Project COO-3243-2, pp. 170-200, USAEC (1973). 


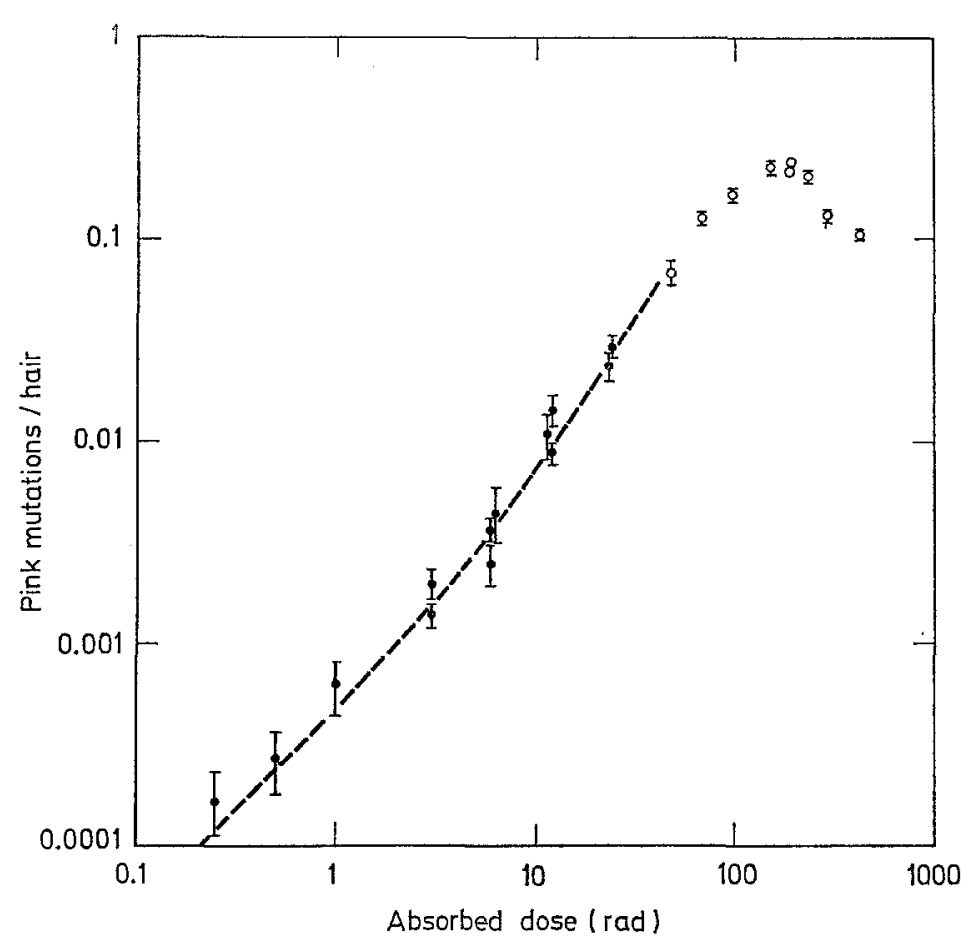

Fig. 1. Yield of colourless mutations in the stamen hairs of Tradescantia as a function of X-ray dose. The points represent the observed yields minus the yield of the controls; the bars represent twice the standard deviations. The observations at low doses (full circles) are used to obtain the least-squares fit to Eq. (5); the curve of the yield minus spontaneous incidence which results from the least-squares fit is given as a broken line

constant slope in the full logarithmic plot. However, even in these cases the analysis in terms of the linear-quadratic model is more meaningful.

\section{Linear Regression Analysis of the Dose-Response Function}

The statistical procedures necessary to derive the least-squares fit and to obtain the joint confidence region for the parameters of response functions such as Eqs. (2) to (4) are well established (see e.g. [2]). It will, however, be useful to discuss the principal numerical steps in a form readily applicable to the typical situations encountered in radiation biology. No attempt will be made to present the full reasoning which underlies the numerical procedure since detailed accounts are found in the literature.

\section{Least-Squares Estimate of the Parameters}

Consider the linear-quadratic relation between effect, $y$, and dose $^{2}$ :

$$
y(D)=a_{1}+a_{2} D+a_{3} D^{2} .
$$

2 The symbol $y$ is used for the effect in order to avoid confusion with the symbol $e$ which will be used for the statistical error. 
The expression is linear in the parameters, $a_{1}, a_{2}$, and $a_{3}$ whose true values are unknown, and have to be estimated from the experimental data. The following considerations will, with minor modifications, apply to all models which are linear in the parameters, regardless how the dose enters the expression.

Assume that a series of doses $D_{v},(v=1, \ldots N)$, is applied in an experiment, and that the observed effect levels at these dose values are $y_{\nu}$. One or several of the $D_{v}$ may have the value zero, i.e. they may represent control groups.

The least-squares problem consists in finding the values of the parameters $a_{1}$, $a_{2}$ and $a_{3}$ which minimize the sum of squares, $\sum_{\nu=1}^{N} e_{\nu}^{2}$, where the $e_{\nu}$ are the differences between the observations, $y_{\nu}$, and the theoretical effect levels, $y\left(D_{\nu}\right)$ :

$$
e_{\nu}=y\left(D_{\nu}\right)-y_{\nu}=a_{1}+a_{2} D+a_{3} D^{2}-y_{\nu} ; \quad(\nu=1, \ldots \mathrm{N})
$$

It is practical to write these relations in matrix form (for details see texts such as [3]). In the following, vectors will be represented by bold-faced small letters, matrices by bold-faced capital letters. Components of vectors or matrices will be indicated by the corresponding letters not in bold face but with indices. Use of the matrix notation will make it clear how the analysis is to be modified for doseresponse functions different from the linear-quadratic form.

If one introduces the vector of errors, $\boldsymbol{e}$, the vector of observations, $\boldsymbol{y}$, and the vector of parameters, $\boldsymbol{a}$ :

$$
\boldsymbol{e}=\left(\begin{array}{c}
e_{1} \\
\vdots \\
\vdots \\
e_{N}
\end{array}\right), \quad \boldsymbol{y}=\left(\begin{array}{c}
y_{1} \\
\vdots \\
\vdots \\
y_{N}
\end{array}\right), \quad \text { and } \quad \boldsymbol{a}=\left(\begin{array}{c}
a_{1} \\
a_{2} \\
a_{3}
\end{array}\right)
$$

and defines the matrix, $\boldsymbol{C}$, of coefficients:

$$
\boldsymbol{C}=\left(\begin{array}{ccc}
1 & D_{1} & D_{1}^{2} \\
\vdots & \vdots & \vdots \\
\vdots & \vdots & \vdots \\
1 & D_{N} & D_{N}^{2}
\end{array}\right)
$$

Then Eq. (6) takes the form:

$$
\boldsymbol{e}=\boldsymbol{C} \boldsymbol{a}-\boldsymbol{y} .
$$

The vector $\boldsymbol{a}$ has to be chosen so that the vector, $\boldsymbol{e}$, of errors has minimum length. The problem can be reduced to the solution of a system of linear equations. Since the mathematics is elementary, and since computer routines are commonly available to derive the solutions $\hat{\boldsymbol{a}}$ and $\hat{\boldsymbol{e}}$ of the minimization problem for a given matrix, $\boldsymbol{C}$, of coefficients and a given vector, $\boldsymbol{y}$, of observations, there is no necessity to list explicit details. The solution is, however, given in Appendix I. The vector $\hat{\boldsymbol{a}}$ is called the estimate of the parameters.

Up to this point it has been assumed that all the observations, $y_{v}$, have the same weight. This is in general not justified because the standard deviations, $\sigma_{\nu}$, associated with the observations, $y_{\nu}$, may be different. Instead of $\sum_{\nu=1}^{N} e_{y}^{2}$ one must therefore minimize the weighted sum of squares of errors, $\sum_{\nu=1}^{N}\left(e_{v} / \sigma_{\nu}\right)^{2}$. In 
some cases it is possible to obtain estimates of the values $\sigma_{\nu}$ from the experimental observations. For instance, in growth reduction studies with Vicia faba, groups of seedlings are exposed to different doses; one can in this case estimate both the standard deviation of the effect and the mean effect in each group $[4,5]$. In other cases such as the scoring of certain mutations or in cellular survival studies one determines merely the number of cells in an exposed group of cells which show the effect. The variance $\sigma_{v}^{2}$ can then be estimated as $n_{v}\left(1-n_{v} / N_{v}\right)$, where $n_{v}$ is the number of cells which show the effect and $N_{v}$ is the total number of cells in the group. When one deals with small rates, $n_{v} / N_{v}$, one can estimate $\sigma_{v}^{2}$ by $n_{v}$. These approximations for the variance of the individual points are based on the assumption that one deals with a binomial distribution or a Poisson distribution of $n_{v}$. This may not always be strictly correct, and the actual variance may be larger than the predicted values. The estimates chosen for $\sigma_{v}$ are, however, acceptable as long as they are proportional to the actual variances, $\sigma_{y}$; the essential requirement is merely that the individual points be given their correct relative weights.

To achieve the proper correction one has to substitute the terms $e_{\nu}, y_{v}$, and $C_{\nu x}$ in Eq. (9) by the expression $e_{v} / \sigma_{v}, y_{v} / \sigma_{\nu}$ and $C_{v x} / \sigma_{v}$. In the following it will be assumed that this normalization is applied; however for simplicity of notation the symbols $e_{v}, y_{p}$, and $C_{v x}$ will be retained for the modified terms.

\section{The Joint Confidence Region}

It is not uncommon that estimates of the parameters of a response function are determined and their standard errors or confidence ranges are given separately. This can, however, be misleading insofar as the estimates of the parameters are not independent. In the linear-quadratic equation, for example, a larger estimate of one of the parameters would imply a decrease of the optimum estimates of the other two parameters. One can ask for that region in the space of the three parameters $a_{1}, a_{2}$, and $a_{3}$ which corresponds to a given value of $\sum e_{v}^{2}$ larger than the minimum value obtainable with optimum fit. These regions are ellipsoids as can be seen in the following way.

Assume that the least-squares estimates of the parameters are designated by $\hat{a}_{\mathrm{k}}$ and that the corresponding sum of least squares, $\sum \hat{e}_{p}^{2}$ is designated by $\hat{s}$;

$$
\|\boldsymbol{C} \hat{\boldsymbol{a}}-\boldsymbol{y}\|^{2}=\hat{s}^{\star} \text {. }
$$

In order to obtain the region in the space of the parameters which correspond to a larger sum of squares, $s+\hat{s}$, one must solve the equation:

$$
\|\boldsymbol{C a}-\boldsymbol{y}\|^{\mathbf{2}}=\hat{s}+s .
$$

One can show (see Appendix II) that this equation is equivalent to:

$$
\|\boldsymbol{C} \alpha\|^{2}=s
$$

where the components of $\alpha, \alpha_{x}=a_{\mathrm{k}}-\hat{a}_{\mathrm{k}}$, are the deviations of the parameters from their estimated values. This is the equation of an ellipsoid centered at $\hat{\boldsymbol{a}}, \boldsymbol{i} . e$. at the least squares estimate of the parameters. The shape and orientation of the

$\star$ The double brackets || denote the norm of a vector. The term on the left-hand side of the equation is therefore the square of the length of the vector $(\boldsymbol{C a}-\boldsymbol{y})$, and $\Sigma \hat{e}_{\nu}^{2}$ could also be written as $\|\hat{e}\|^{2}$. 
ellipsoid is determined by $\boldsymbol{C}$ and the size by $s$. The equation can be written in the normalized form:

$$
\alpha^{\prime} B \alpha=1^{\star \star}
$$

where the symmetric $(3 \times 3)$-matrix $\boldsymbol{B}$ is defined as:

$$
\boldsymbol{B}=\frac{1}{s} \boldsymbol{C}^{\prime} \boldsymbol{C}
$$

From Eq. (13) one readily derives the major axes of the ellipsoid of the three parameters $a_{1}, a_{2}$, and $a_{3}$, or obtains the plot of the ellipse which results if one considers only the two dose coefficients $\alpha_{2}$ and $a_{3}$. This will be dealt with in the next section where numerical results will be given for the data of Fig. 1 . First it will be necessary to consider how the value of $s$ is determined which corresponds to a given level of statistical significance.

The determination of the joint confidence region is based on the assumption that the theoretical expression used to fit the data is valid and that therefore the errors are entirely due to statistical fuctuations and not to systematic deviations of the experimental results. Moreover one assumes that the errors, $e_{p}$, are independent and normally distributed.

As a first step, before one considers the confidence region of the parameters, one must therefore check whether the theoretical model is indeed acceptable. There are various possible tests which can be applied to check the adequacy of a model; all these tests are based on an examination of the residuals $\hat{e}_{v}=\hat{y}_{\nu}-y_{v}$. The general method is to apply suitable non-parametric tests of randomness to the set of values $\hat{e}_{v}$. If, however, the variances, $\sigma_{v}^{2}$, of the observations are known, the simplest test of adequacy is the comparison of the weighted sum of least squares, $\hat{s}=\sum_{v=1}^{N} \hat{e}_{\nu}^{2} / \sigma_{\nu}^{2}$, with the critical value of the statistic $\chi^{2}$ with $(N-M)$ degrees of freedom. If the test of adequacy of the theoretical model does not lead to its rejection, one can proceed to the second step and determine the scalar $s$ and the resulting region of confidence of the parameters.

There are two different methods for the determination of the value of $s$. The choice of the method depends on whether the variances, $\sigma_{v}^{2}$, themselves or only the relative weights of the individual observations are known.

If one knows only the relative weight of the individual observations, one has to use the fact that $s / \hat{s}$ is distributed according to Fisher's $F$-distribution with $M$ degrees of freedom for the numerator and $(N-M)$ degrees of freedom for the denominator. The joint region of the parameters with the confidence level $p$ is obtained by using the following value of $s$ in Eqs. (13) and (14):

$$
s=\hat{s} \frac{M}{N-M} F(M, N-M, p) \text {. }
$$

$N$ is the number of observations (dose values), $M$ the number of parameters, and $F(M, N-M, p)$ the critical value of Fisher's $F$-distribution on the level $p$ of confidence.

In the case of the linear-quadratic model $M$ is equal to 3 , but the formulae apply to the general case of a linear model of arbitrary order $M$. Tables of critical

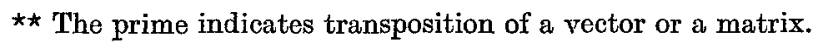


values of $F(M, L, p)$ for various degrees of freedom, $M$ and $L$, and for different confidence levels $p$ are found in the literature $[2,9]$.

If, as in the example of Fig. 1, the values $\sigma_{p}^{2}$ are known, $s$ is distributed according to the statistic $\chi^{2}$ with $M$ degrees of freedom. The value of $s$ defining the joint confidence region on the level $p$ is then given by:

$$
s=\chi_{M}^{2}(p) \text {. }
$$

This leads to an ellipsoid concentric, coaxial, and similar to the one obtained on the basis of Fisher's $F$-distribution; but usually this ellipsoid is somewhat smaller. This reflects the fact that one has more information in this case than in the previous one where only the relative values of the variances, $\sigma_{v}^{2}$, are known.

\section{Numerical Results and Generalization to Non-Linear Cases}

The least-squares fit of Eq. (5) to the data of Fig. 1 results in the sum $\hat{s}=14.7$. This is less than the $95 \%$-value $\chi_{N-3}^{2}=19.7$ for $N=14$. The model can therefore be accepted.

Table 1 represents the result of the least-squares fit and the solution of Eq. (13). In order to define the resulting ellipsoid of the possible values of the parameters in concise but explicit form, the outer points of its main axes are given in addition to the center $\hat{\boldsymbol{a}}$. These values are readily obtained from Eq. (13) by computing the eigen-vectors of the matrix $\boldsymbol{B}$ (see Appendix III).

Also given in Table 1 are the $95 \%$-values and $50 \%$-values of the statistics $\chi_{3}^{2}$ and $3 /(N-3) F(3, N-3)$. The numerical results are based on the statistic $\chi_{3}^{2}$, i.e. Eq. (13) has been solved with the value $s=7.82$. If one were to use the value $s=14.4$ resulting from Fisher's distribution, one would obtain a confidence region with linear dimensions increased by a factor 1.36 .

While Table 1 contains the full numerical results, one may want to present these results in simpler form. In order to do this, one can eliminate the parameter $a_{1}$, i.e. the spontaneous incidence of the mutations, and merely consider the confidence region of the dose coefficients $a_{2}$ and $a_{3}$. This confidence region corresponds to the projection of the ellipsoid of the three parameters into the plane of the two parameters $a_{2}$ and $a_{3}$. But its size is somewhat smaller because one must set $s$ equal to $\chi_{2}^{2}$ or $2 /(N-3) \cdot F(2, N-3) \cdot \hat{s}$, since $M$ is equal to 2 instead of 3 . The resulting figure is an ellipse as shown in Fig. 2. The inner ellipse, drawn as a broken line, corresponds to the $50 \%$-level of the statistic $\chi_{2}^{2}$, and can therefore be considered as the region of standard deviation of the parameters $a_{2}$ and $a_{3}$. How the matrix of coefficients for the ellipse is derived from the matrix $\boldsymbol{B}$ is pointed out in Appendix IV. One should note that one would obtain regions which can be somewhat too small, if one were to derive the intersection of the ellipsoid with the plane $a_{1}=\hat{a}_{1}$ instead of its projection onto the plane of $a_{2}$ and $a_{3}$. One should further note that the form of the ellipsoids and ellipses, i.e. the orientation of the axes, depends on the choice of the unit of dose. For a suitable plot it is desirable that the values of the different parameters are of the same order of magnitude. A proper choice of the unit for the numerical calculation is therefore a dose for which the linear and the quadratic components in the response function are about equal. As a value close to the bend of the curve in Fig. 1 the unit of 10 rad has been chosen for the computations in the present example. 
Table 1. Coordinates of the $95 \%$-confidence region of the parameters $a_{1}, a_{2}$ and $a_{3}$ in Eq. (5) applied to the data of Fig. 1 and critical values of the statistics $\chi^{2}$ and $F$

$95 \%$-confidence ellipsoid of the parameters based on the $\chi^{2}$-statistic

\begin{tabular}{llll}
\hline & $a_{1}$ & $\begin{array}{l}a_{2} \\
\left(\mathrm{rad}^{-1}\right)\end{array}$ & $\begin{array}{l}a_{3} \\
\left(\mathrm{rad}^{-2}\right)\end{array}$ \\
Center $\left(\hat{a}_{i}\right)$ & $7.17 \cdot 10^{-4}$ & $4.03 \cdot 10^{-4}$ & $3.05 \cdot 10^{-5}$ \\
\hline End points of axes & 8.14 & 4.03 & 3.05 \\
& 6.20 & 4.03 & 3.05 \\
& 7.00 & 4.64 & 3.69 \\
& 7.35 & 3.42 & 2.41 \\
& 6.90 & 5.91 & 1.26 \\
& 7.45 & 2.15 & 4.84 \\
\hline
\end{tabular}

$N=14 ; M=3 ; \widehat{s}=\|\widehat{\varepsilon}\|^{2}=14.7 ; \chi^{2}{ }_{11}(0.95)=19.7 ;$ $\chi^{2}{ }_{11}(0.5)=10.3$.

Critical values of $F$ and $\chi^{2}$ and corresponding values of $s$ : $\chi^{2}{ }_{3}(0.95)=7.82 ; s=7.82$.

$3 / 11 \cdot F(3,11,0.95)=0.98 ; s=14.4$.

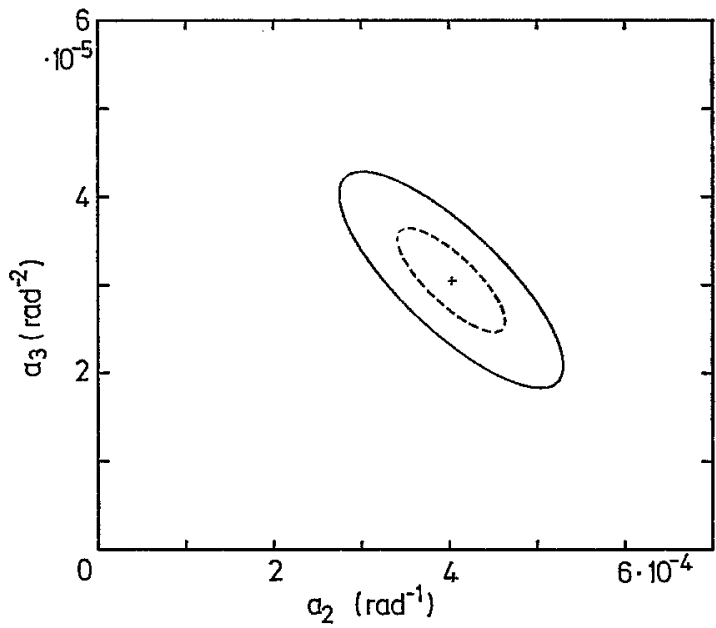

Fig. 2. 95\%-confidence region for the parameters $a_{2}$ and $a_{3}$ of the linear-quadratic model, Eq. (5), applied to the data of Fig. 1. The broken ellipse delineates the region of standard deviation

The studies with Tradescantia permit relatively high statistical accuracy even at low doses, as is apparent from Fig. 2. This is due to the nature of the experimental system in which a small number of affected cells can be detected among an extremely large number of exposed cells. In other systems to which the method has been applied $[4,5,13]$ the relative size of the confidence regions has been larger. The standard deviations, $\sigma_{v}$, indicated in Fig. 1 are derived under the assumption that one deals with Poisson distributions of the number of affected cells at small doses. This is not necessarily a valid assumption, since at each dose, $D_{v}$, one 
scores a large number of cells in a limited number of plants; sensitivity variations between plants or interdependence of the mutagenetic events in neighboring cells in the same stamen hairs could lead to a variance which exceeds that of a Poisson distribution. Whether this is indeed the case cannot be decided in the present example, because the least-squares sum, $\hat{s}$, although larger than the $50 \%$-value is considerably smaller than the $95 \%$-value of $\chi_{N-3}^{2}$. For this reason the validity of the standard deviations depicted in Fig. 1 has been accepted, and the analysis has been based on the $\chi^{2}$-statistic.

From the estimates and confidence regions for $a_{2}$ and $a_{3}$ one obtains the corresponding range of the quantities $k=a_{3}$ and $\zeta=a_{2} / a_{3}$ in Eq. (2). The microdosimetric implications of the observed value of $\zeta$ and its confidence range is not the object of the present study, nor will the influence of saturation be considered which leads to deviations from the linear-quadratic model at higher doses.

The mathematical procedure, here applied to the linear-quadratic model, can be modified to apply to other cases. In the case of Eq. (4) which has been used for survival curves of mammalian cells [14] or growth reduction studies in plants [4, 5] no modifications are necessary, but one must be sure to equate the values $\sigma_{\nu}$ with the standard deviation of the logarithm of the observed variable and not with the standard deviation of its absolute value. A simplification occurs in those cases where one deals with only two parameters, i.e. if the term which is quadratic in dose can be neglected with densely ionizing radiations. The mathematical problem of the derivation of the confidence region then reduces to a problem in two dimensions. The modifications in the formalism are obvious.

More complicated are those models which are non-linear in the unknown parameters. One example is the model defined by the relation:

$$
y(D)=a_{1}+a_{2} D^{n} .
$$

In this case the equations for the least-squares problem can be readily established; but their analytical solution is difincult. The simplest method is therefore to use a computer and perform the least-squares fit for a series of fixed values of $n$. In this way the least-squares estimate of $n$ can be determined. The same technique can be used in order to obtain the confidence region section by section.

Frequently it is possible to linearize a model by considering a suitable function of $y(D)$ instead of $y(D)$ itself. This can also be done in the case of Eq. (17). Since the parameters of interest in this equation are $a_{2}$ and $n$ rather than $a_{1}$, the response function can be rewritten in the form:

$$
\ln \left[y(D)-a_{1}\right]=\ln a_{2}+n \ln D .
$$

With this modification the model is linear in the two parameters $\ln a_{2}$ and $n$, and the confidence region of these parameters can be readily obtained for a series of values of $a_{1}$. Similar considerations can be applied to other dose-response functions.

\section{Appendix}

The following considerations, although related to the particular example discussed in the present context, are formulated so that they apply to the general case of $M$ linear parameters. The derivations are given in compact form suitable for reference. More elementary explanations are found in the literature (see e.g. [2]). 


\section{Solution of the Least-Squares Problem}

Eq. (11) has the form:

$$
\boldsymbol{e}=\boldsymbol{C a}-\boldsymbol{y} .
$$

If one considers all vectors $\boldsymbol{a}$, then the corresponding vectors $\boldsymbol{C a}$ define an $\boldsymbol{M}$ dimensional subspace, $W$, in the $N$-dimensional space of the vectors $\boldsymbol{y}$ of observations. The least-squares solution, $\hat{\boldsymbol{a}}$, is the vector for which the length of the vector $\boldsymbol{e}$ is minimum. Therefore $\boldsymbol{e}$ must be orthogonal to $W$. Accordingly its scalar products with the columns of $\boldsymbol{C}$, which are linearly independent vectors in $W$, must be zero:

$$
\boldsymbol{C}^{\prime}(\boldsymbol{C} \hat{\boldsymbol{a}}-\boldsymbol{y})=\mathbf{0} .
$$

Therefore the solution of the least-squares problem is:

$$
\hat{\boldsymbol{a}}=\left(\boldsymbol{C}^{\prime} \boldsymbol{C}\right)^{-\mathbf{1}} \boldsymbol{C}^{\prime} \boldsymbol{y} \text {. }
$$

\section{Equation of the Confidence Region}

Eqs. (10) and (11) have the form:

and:

$$
\|\boldsymbol{C} \hat{\boldsymbol{a}}-\boldsymbol{y}\|^{\mathbf{2}}=\hat{\boldsymbol{s}}
$$

Furthermore one has:

$$
\|\boldsymbol{C a}-\boldsymbol{y}\|^{2}=s+\hat{s} .
$$

$$
\boldsymbol{C}(\boldsymbol{a}-\hat{\boldsymbol{a}})=(\boldsymbol{C a}-\boldsymbol{y})-(\boldsymbol{C} \hat{a}-\boldsymbol{y})
$$

where $\boldsymbol{C}(\boldsymbol{a}-\hat{\boldsymbol{a}})$ is a vector of $W$, while $(\boldsymbol{C} \hat{\boldsymbol{a}}-\boldsymbol{y})$ is orthogonal to $W$. Therefore:

and:

$$
\|C(a-\hat{a})\|^{2}=\|C a-y\|^{2}-\|C \hat{a}-y\|^{2}
$$

$$
\|\boldsymbol{C}(\boldsymbol{a}-\hat{\boldsymbol{a}})\|^{2}=s .
$$

This is the Eq. (12) of the ellipsoid.

\section{Half Axes of the Ellipsoid}

According to Eq. (13) the equation of the centered ellipsoid is:

$$
\boldsymbol{\alpha}^{\prime} \boldsymbol{B} \alpha=1 \text {. }
$$

The major axes have the property that they have the same direction as the normal vector at their intersection points with the surface of the ellipsoid. The normal vector at a point $\boldsymbol{\alpha}$ of the surface is $\boldsymbol{B} \alpha$, therefore if $\alpha_{i}$ is the vector defining the $i$-th half axis:

$$
\boldsymbol{B} \alpha_{i}=\lambda_{i} \alpha_{i} .
$$

This is the definition of an eigen-vector of $\boldsymbol{B}$, and $\lambda_{i}$ is its eigen-value. Inserting $\alpha_{i}$ into Eq. (27) one obtains:

$$
\alpha_{i}^{\prime} \boldsymbol{B} \alpha_{i}=\lambda_{i}\left\|\alpha_{i}\right\|^{2}=1 .
$$

Thus the major axes have the direction of the eigen-vectors of $\boldsymbol{B}$ and the eigenvalues are the inverse squares of the length of the half axes.

\section{Projection of the Ellipsoid}

Assume that the centered $M$-dimensional ellipsoid is projected along the direction of the first coordinate vector, $\boldsymbol{u}_{1}$. The points, $\boldsymbol{a}$, on the surface of the 
ellipsoid which form the contour of the projection have normal vectors, $\boldsymbol{B} \boldsymbol{a}$, orthogonal to $\boldsymbol{u}_{\mathbf{1}}$. Therefore the first component of $\boldsymbol{B} \boldsymbol{a}$ is zero:

and

$$
\boldsymbol{u}_{1}^{\prime} \boldsymbol{B} a=\sum_{k=1}^{M} B_{1 k} a_{k}=0
$$

$$
a_{1}=-\sum_{k=2}^{M} \frac{B_{1 k}}{B_{11}} a_{k}
$$

Therefore the set of points $a$ is obtained from the relation:

$$
\boldsymbol{a}=\boldsymbol{T r}
$$

where $\boldsymbol{T}$ is a matrix of $M$ rows and ( $M-1$ ) columns which contains the terms $B_{1}, \mathbf{k}+1 / B_{11}$ as the $k$-th element of its first row and has the form of a unit diagonal matrix below the first row. The vector $\boldsymbol{r}$ is the truncated form of $\boldsymbol{\alpha}$ which results if one eliminates its first component $a_{1}$. In the special case of $M=3$ one has:

$$
\boldsymbol{T}=\left(\begin{array}{cc}
-\frac{B_{12}}{B_{11}}-\frac{B_{13}}{B_{11}} \\
1 & 0 \\
0 & 1
\end{array}\right) \quad \text { and } \quad r=\left(\begin{array}{c}
a_{2} \\
a_{3}
\end{array}\right)
$$

The equation of the (M-1)-dimensional projection figure is therefore:

$$
\boldsymbol{r}^{\prime} \boldsymbol{T}^{\prime} \boldsymbol{B} \boldsymbol{T} \boldsymbol{r}=1 \text {. }
$$

This means that the $(M-1)$-dimensional projection of an $M$-dimensional ellipsoid defined by a matrix $\boldsymbol{B}$ is again an ellipsoid and that it is defined by the matrix $\boldsymbol{T}^{\prime} \boldsymbol{B} \boldsymbol{T}$. The result can be easily generalized to the elimination of more than one parameter. As pointed out earlier, the value of $s$ which enters the definition of $\boldsymbol{B}$ in Eq. (14) must correspond to the actual number of parameters under consideration.

Acknowledgement. This investigation was supported by Contract AT-(30-1)-3243 from the U.S. Atomic Energy Commission and Research Grant No. CA-12536 from the National Cancer Institute. The authors are indebted to Dr. William Gross for valuable help in the preparation of the manuscript.

\section{References}

1. Conger, A. D.: The fate of metaphase aberrations. Radiation Botan. 5, 81-96 (1965)

2. Draper, N. R., Smith, H.: Applied regression analysis. Wiley 1966

3. Faddeeva, V. N.: Computational methods of linear algebra. Dover 1959

4. Hall, E. J., Kellerer, A. M.: The biophysical properties of 3.9-GeV nitrogen ions. Radiation Research 55, 422-430 (1973)

5. Hall, E. J., Rossi, H. H., Kellerer, A. M., Goodman, L., Marino, S.: Radiobiological studies with monoenergetic neutrons. Radiation Research 54, 431-443 (1973)

6. Hug, O., Kellerer, A. M. : Stochastik der Strahlenwirkung. Berlin-Heidelberg-New York: Springer 1966

7. Kellerer, A. MI., Rossi, H. H.: The theory of dual radiation action. In: Current Topics in Radiation Research 8, 85-158 (1972)

8. Lea, D. W.: Actions of radiation on living cells. Cambridge: University Press 1946

9. Pearson, E. S., Hartley, H. D.: Biometrika tables for statisticians, Vol. 1. Cambridge: University Press 1958

10. Read, J.: The induction of chromosome exchange aberrations by ionizing radiations; the "site concept". Intern. J. Radiation Biol. 9, 53-65 (1965) 
11. Rossi, H. H.: Specification of radiation quality. Radiation Research 10, 522-531 (1959)

12. Rossi, H. H.: Energy distribution in the absorption of radiation. Advances in Biol. and Med. Phys. 11, 27-85 (1967)

13. Rossi, H. H., Kellerer, A. M.: The validity of risk estimates of leukemia based on Japanese data. Radiat. Res. (in press)

14. Sinclair, W. K.: Radiation survival in synchronous and asynchronous Chinese hamster cells in vitro. In: Biophysical aspects of radiation quality, pp. 39-54. IAEA, Vienna 1968

15. Sparrow, A. H., Underbrink, A. G., Rossi, H. H.: Mutations induced in Tradescantia by small doses of X-rays and neutrons: analysis of dose-response curves. Science 176, 916-918 (1972)

16. Wolff, S.: Interpretations of induced chromosome breakage and rejoining. Radiat. Res. Suppl. 1, 453-462 (1959)

Prof. Dr. A. M. Kellerer

Radiological Research Laboratories

Department of Radiology

College of Physicians and Surgeons

of Columbia University

630 West 168th Street

New York, N.Y. 10032 (U.S.A.) 